sono eseguiti 3896 dosaggi anti-HCV su campioni pervenuti al Laboratorio di Patologia Clinica dai vari Reparti o come utenti esterni, utilizzando il kit della Ditta Abbott, su apparecchiatura AxSYM, basato su un metodo immunoenzimatico a cattura di microparticelle.

Risultati. 136 campioni sono risultati positivi al test presentando valori $\mathrm{S} / \mathrm{CO}$ superiori a 3.14 campioni presentavano valori di S/CO superiori a 1 ma inferiori a 3. Tutti i 14 campioni sono stati sottoposti a test di conferma Immunoblot. I risultati hanno evidenziato che il $75 \%$ di essi è risultato negativo, il $20 \%$ ha dato esito indeterminato e solo il $5 \%$ è risultato positivo.

Conclusioni. Si conferma come nella popolazione esista una elevata percentuale di soggetti positivi ai tests di screening anti-HCV e che con l'esclusivo impiego di tali tests non è possibile formulare una diagnosi corretta., per cui il test di screening deve essere, necessariamente, supportato da un test addizionale di conferma, che possa limitare i casi risultati falsi positivi.

\title{
ANTI - HCV : DIAGNOSI DI LABORATORIO
}

\section{Migliozzi A., Cocco M.P.}

Dipartimento di Patologia Clinica - U.O. Medicina di Laboratorio - P.O. S.Maria delle Grazie ASL NA2

Introduzione. La positività anti-HCV è rivelata in primo luogo mediante tecniche ELISA ed è successivamente confermata mediante Immunoblot.

I suddetti tests sono dotati di elevata specificità e sensibilità ma presentano, tuttavia, dei limiti che sono propri della diagnosi di tipo indiretto. Il loro impiego, quindi può fornire reazioni falsamente negative o falsamente positive.

Le prime sono dovute, per lo più al periodo di finestra immunologica del soggetto mentre le seconde possono essere la conseguenza di svariati fattori quali: modalità di conservazione del campione, cross-reattività con altri flavovirus, ipergammaglobulinemia .

Per questi motivi, quindi, il Laboratorio deve confermare la positività anti-HCV mediante tests aggiuntivi di Immunoblot, che acquistano particolare rilevanza nei campioni "borderline".

Scopo del seguente studio è stato quello di valutare la prevalenza, nella popolazione generale, dei soggetti anti-HCV positivi a basso titolo anticorpale, tra 1 e $3 \mathrm{~S} / \mathrm{CO}$ ( valore del campione/valore limite) .

Materiali e metodi. Nel periodo Gennaio-Dicembre 2004 si 